\title{
CG Versus MINRES: An Empirical Comparison
}

\section{David Chin-Lung Fong* and Michael Saunders**}

*ICME, Stanford University, California, USA, Email: david3.14159@gmail.com.

**Systems Optimization Laboratory, Department of Management Science and Engineering, Stanford University, California, USA, Email: saunders@stanford.edu.

ABSTRACT: For iterative solution of symmetric systems $A x=b$, the conjugate gradient method (CG) is commonly used when $A$ is positive definite, while the minimum residual method (MINRES) is typically reserved for indefinite systems. We investigate the sequence of approximate solutions $x_{k}$ generated by each method and suggest that even if $A$ is positive definite, MINRES may be preferable to CG if iterations are to be terminated early. In particular, we show for MINRES that the solution norms $\left\|x_{k}\right\|$ are monotonically increasing when $A$ is positive definite (as was already known for $\mathrm{CG}$ ), and the solution errors $\left\|x^{*}-x_{k}\right\|$ are monotonically decreasing. We also show that the backward errors for the MINRES iterates $x_{k}$ are monotonically decreasing.

KEYWORDS: Conjugate gradient method (CG), Conjugate residual method (CR), Iterative method, Krylov subspace method, Linear equations, Minimum residual method (MINRES), Sparse matrix, Trust-region method.

\section{مقارنة تجريبية بين طريقتي التترج المترافقة والخطأ المتبقي الأصغر ديفيد تشن-لونج فونج و ميخائيل سوندرز}

\footnotetext{
ملخص: من المعتاد استخدام طريقة التدرج المنر افقة لحل جملة من المعادلات الخطية المتناظرة

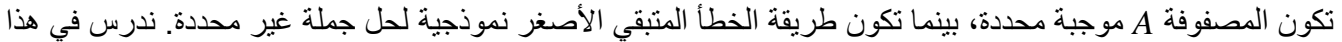

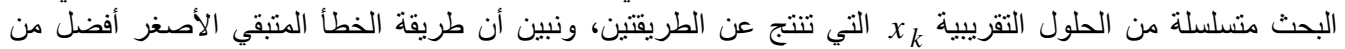
طريقة التدرج المتر افقة، حتى عندما تكون المصفوفة A

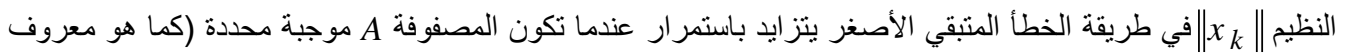
في حالة طريقة التدرج المتر افقة) وأن أخطاء الحل|| |x طريقة الخطأ المنبقي الأصغر نتناقص باستمرار.
} 


\section{CG VERSUS MINRES: AN EMPIRICAL COMPARISON}

\section{Introduction}

$\mathbf{T}$

The conjugate gradient method (CG) (Hestenes and Stiefel, 1952) and the minimum residual method (MINRES) (Paige and Saunders, 1975) are both Krylov subspace methods for the iterative solution of symmetric linear equations $A x=b$. CG is commonly used when the matrix $A$ is positive definite, while MINRES is generally reserved for indefinite systems (van der Vorst, 2003, p85). We re-examine this wisdom from the point of view of early termination on positive-definite systems.

We assume that the system $A x=b$ is real with $A$ symmetric positive definite (spd) and of dimension $n \times n$. The Lanczos process (Lanczos, 1950) with starting vector $b$ may be used to generate the $n \times k$ matrix $V_{k} \equiv\left(v_{1} v_{2} \cdots v_{k}\right)$ and the $(k+1) \times k$ Hessenberg tridiagonal matrix $\underline{T_{k}}$ such that $A V_{k}=V_{k+1} \underline{T_{k}}$ for $k=1,2, \cdots, \ell$ and $A V_{\ell}=V_{\ell} T_{\ell}$ for some $\ell \leq n$, where the columns of $V_{k}$ form a theoretically orthonormal basis for the $k$ th Krylov subspace $\mathcal{K}_{k}(A, b) \equiv \operatorname{span}\left\{b, A b, A^{2} b, \cdots, A^{k-1} b\right\}$, and $T_{\ell}$ is $\ell \times \ell$ and tridiagonal. Approximate solutions within the $k$ th Krylov subspace may be formed as $x_{k}=V_{k} y_{k}$ for some $k$-vector $y_{k}$. As shown in (Paige and Saunders, 1975), three iterative methods CG, MINRES, and SYMMLQ may be derived by choosing $y_{k}$ appropriately at each iteration. CG is well defined if $A$ is spd, while MINRES and SYMMLQ are stable for any symmetric nonsingular $A$.

As noted by Choi (2006), SYMMLQ can form an approximation $x_{k+1}=V_{k+1} y_{k+1}$ in the (k+1)th Krylov subspace when CG and MINRES are forming their approximations $x_{k}=V_{k} y_{k}$ in the $k$ th subspace. It would be of future interest to compare all three methods on spd systems, but for the remainder of this paper we focus on CG and MINRES.

With different methods using the same information $V_{k+1}$ and $\underline{T_{k}}$ to compute solution estimates $x_{k}=V_{k} y_{k}$ within the same Krylov subspace (for each $k$ ), it is commonly thought that the number of iterations required will be similar for each method, and hence CG should be preferable on spd systems because it requires somewhat fewer floating-point operations per iteration. This view is justified if an accurate solution is required (stopping tolerance $\tau$ close to machine precision $\varepsilon$ ). We show that with looser stopping tolerances, MINRES is sure to terminate sooner than CG when the stopping rule is based on the backward error for $x_{k}$, and by numerical examples we illustrate that the difference in iteration numbers can be substantial.

\subsection{Notation}

We study the application of CG and MINRES to real symmetric positive-definite (spd) systems $A x=b$. The unique solution is denoted by $x *$. The initial approximate solution is $x_{0} \equiv 0$, and $r_{k} \equiv b-A x_{k}$ is the residual vector for an approximation $x_{k}$ within the $k$ th Krylov subspace. For a vector $v$ and matrix $A,\|v\|$ and $\|A\|$ denote the 2-norm and the Frobenius norm respectively, and $A \succ 0$ indicates that $A$ is spd.

\section{Minimization properties of Krylov subspace methods}

With exact arithmetic, the Lanczos process terminates with $k=\ell$ for some $\ell \leq n$. To ensure that the approximations $x_{k}=V_{k} y_{k}$ improve by some measure as $k$ increases toward $\ell$, the Krylov solvers minimize some convex function within the expanding Krylov subspaces (Freund et al., 1992). 


\section{DAVID CHIN-LUNG FONG and MICHAEL SAUNDERS}

\subsection{CG}

When $A$ is spd, the quadratic form $\phi(x) \equiv \frac{1}{2} x^{T} A x-b^{T} x$ is bounded below, and its unique minimizer solves $A x=b$. A characterization of the $\mathrm{CG}$ iterations is that they minimize the quadratic form within each Krylov subspace (Freund et al., 1992; Meurant, 2006, §2.4; Watkins, 2010, §8.8-§8.9):

$$
x_{k}^{C}=V_{k} y_{k}^{C}, \quad \text { where } \quad y_{k}^{C}=\arg \min \phi\left(V_{k} y\right) .
$$

With $b=A x *$ and $2 \phi\left(x_{k}\right)=x_{k}^{T} A x_{k}-2 x_{k}^{T} A x^{*}$, this is equivalent to minimizing the function $\left\|x *-x_{k}\right\|_{A} \equiv\left(x *-x_{k}\right)^{T} A\left(x *-x_{k}\right)$, known as the energy norm of the error, within each Krylov subspace. For some applications, this is a desirable property (Steihaug, 1983; Sun, 2003; Arioli, 2004; Meurant, 2006; Watkins, 2010).

\subsection{MINRES}

For nonsingular (and possibly indefinite) systems, the residual norm was used in (Paige and Saunders, 1975) to characterize the MINRES iterations:

$$
x_{k}^{M}=V_{k} y_{k}^{M}, \quad \text { where } \quad y_{k}^{M}=\underset{y}{\arg \min }\left\|b-A V_{k} y\right\| \text {. }
$$

Thus, MINRES minimizes $\left\|r_{k}\right\|$ within the $k$ th Krylov subspace. This was also an aim of Stiefel's Conjugate Residual method (CR) (Stiefel, 1955) for spd systems (and of Luenberger's extensions of CR to indefinite systems (Luenberger, 1969, 1970)). Thus, CR and MINRES must generate the same iterates on spd systems. We use this connection to prove that $\left\|x_{k}\right\|$ increases monotonically when MINRES is applied to an spd system.

\subsection{CG and CR}

The two methods for solving spd systems $A x=b$ are summarized in Table 1 . The first two columns are pseudocodes for $\mathrm{CG}$ and $\mathrm{CR}$ with iteration number $k$ omitted for clarity; they match our Matlab implementations. Note that $q=A p$ in both methods, but it is not computed as such in CR. Termination occurs when $r=0$ $(\Rightarrow \rho=\beta=0)$.

To prove our main result we need to introduce iteration indices; see column 3 of Table 1 . Termination occurs when $r_{k}=0$ for some index $k=\ell \leq n\left(\Rightarrow \rho_{\ell}=\beta_{\ell}=0, \quad r_{\ell}=s_{\ell}=p_{\ell}=q_{\ell}=0\right)$. Note: This $\ell$ is the same as the $\ell$ at which the Lanczos process theoretically terminates for the given $A$ and $b$.

Theorem 2.1 The following properties hold for Algorithm CR:

(a) $q_{i}^{T} q_{j}=0(i \neq j)$

(b) $r_{i}^{T} q_{j}=0(i \geq j+1)$

Proof. Given in Luenberger (1970, Theorem 1).

Theorem 2.2 The following properties hold for Algorithm CR:

(a) $\alpha_{i} \geq 0$

(b) $\beta_{i} \geq 0$

(c) $p_{i}^{T} q_{j} \geq 0$ 
(d) $p_{i}^{T} p_{j} \geq 0$

(e) $x_{i}^{T} p_{j} \geq 0$

(f) $r_{i}^{T} p_{j} \geq 0$

\section{Proof.}

(a) Here we use the fact that $A$ is spd. The inequalities are strict until $i=\ell$ (and $r_{\ell}=0$ ).

$$
\begin{aligned}
& \rho_{i}=r_{i}^{T} s_{i}=r_{i}^{T} A r_{i} \geq 0 \quad(A \succ 0) \\
& \alpha_{i}=\rho_{i-1} /\left\|q_{i-1}\right\|^{2} \geq 0
\end{aligned}
$$

(b) And again:

$$
\beta_{i}=\rho_{i} / \rho_{i-1} \geq 0 \quad(\text { by }(2))
$$

(c) Case I: $i=j$

$$
p_{i}^{T} q_{i}=p_{i}^{T} A p_{i} \geq 0 \quad(A \succ 0)
$$

Case II: $i-j=k>0$

$$
\begin{array}{rlr}
p_{i}^{T} q_{j} & =p_{i}^{T} q_{i-k}=r_{i}^{T} q_{i-k}+\beta_{i} p_{i-1}^{T} q_{i-k} \\
& =\beta_{i} p_{i-1}^{T} q_{i-k} \quad \text { (by Theorem 2.1(b)) } \\
& \geq 0,
\end{array}
$$

where $\beta_{i} \geq 0$ by (b) and $p_{i-1}^{T} q_{i-k} \geq 0$ by induction as $(i-1)-(i-k)=k-1<k$.

Case III: $j-i=k>0$

$$
\begin{aligned}
p_{i}^{T} q_{j} & =p_{i}^{T} q_{i+k}=p_{i}^{T} A p_{i+k} \\
& =p_{i}^{T} A\left(r_{i+k}+\beta_{i+k} p_{i+k-1}\right) \\
& =q_{i}^{T} r_{i+k}+\beta_{i+k} p_{i}^{T} q_{i+k-1} \\
& =\beta_{i+k} p_{i}^{T} q_{i+k-1} \quad \text { (by Theorem 2.1(b)) } \\
& \geq 0,
\end{aligned}
$$

where $\beta_{i+k} \geq 0$ by (b) and $p_{i}^{T} q_{i+k-1} \geq 0$ by induction as $(i+k-1)-i=k-1<k$.

(d) At termination, define $\mathcal{P} \equiv \operatorname{span}\left\{p_{0}, p_{1}, \cdots, p_{\ell-1}\right\} \quad$ and $\mathcal{Q} \equiv \operatorname{span}\left\{q_{0}, \cdots, q_{\ell-1}\right\}$. By construction, $\mathcal{P}=\operatorname{span}\left\{b, A b, \cdots, A^{\ell-1} b\right\}$ and $\mathcal{Q}=\operatorname{span}\left\{A b, \cdots, A^{\ell} b\right\}$ (since $q_{i}=A p_{i}$ ). Again by construction, $x_{\ell} \in \mathcal{P}$, and since $r_{\ell}=0$ we have $b=A x_{\ell} \Rightarrow b \in \mathcal{Q}$. We see that $\mathcal{P} \subseteq \mathcal{Q}$. By Theorem 2.1(a), $\left\{q_{i} /\left\|q_{i}\right\|\right\}_{i=0}^{\ell-1}$ forms an orthonormal basis for $\mathcal{Q}$. If we project $p_{i} \in \mathcal{P} \subseteq \mathcal{Q}$ onto this basis, we have

$$
p_{i}=\sum_{k=0}^{\ell-1} \frac{p_{i}^{T} q_{k}}{q_{k}^{T} q_{k}} q_{k},
$$

where all coordinates are non-negative from (c). Similarly for any other $p_{j}, j<\ell$. Therefore $p_{i}^{T} p_{j} \geq 0$ for any $i, j<\ell$.

(e) By construction, 


\section{DAVID CHIN-LUNG FONG and MICHAEL SAUNDERS}

$$
x_{i}=x_{i-1}+\alpha_{i} p_{i-1}=\cdots=\sum_{k=1}^{i} \alpha_{k} p_{k-1} \quad\left(x_{0}=0\right) .
$$

Therefore $x_{i}^{T} p_{i} \geq 0$ by (d) and (a).

(f) Note that any $r_{i}$ can be expressed as a sum of $q_{i}$ :

$$
\begin{aligned}
r_{i} & =r_{i+1}+\alpha_{i+1} q_{i} \\
& =\cdots \\
& =r_{l}+\alpha_{l} q_{l-1}+\cdots+\alpha_{i+1} q_{i} \\
& =\alpha_{l} q_{l-1}+\cdots+\alpha_{i+1} q_{i} .
\end{aligned}
$$

Thus we have

$$
r_{i}^{T} p_{j}=\left(\alpha_{l} q_{l-1}+\cdots+\alpha_{i+1} q_{i}\right)^{T} p_{j} \geq 0,
$$

where the inequality follows from (a) and (c).

Table 1. Pseudocode for algorithms CG and CR

\begin{tabular}{l} 
CG \\
\hline Initialize \\
$x=0, r=b$ \\
$\rho=\|r\|^{2}, p=r$ \\
Repeat \\
$\quad q=A p$ \\
$\alpha=\rho / p^{T} q$ \\
$\quad x \leftarrow x+\alpha p$ \\
$r \leftarrow r-\alpha q$ \\
$\quad$ \\
$\quad \bar{\rho}=\rho, \rho=r^{T} r$ \\
$\beta=\rho / \bar{\rho}$ \\
$p \leftarrow r+\beta p$
\end{tabular}

\begin{tabular}{l} 
CR \\
\hline Initialize \\
$x=0, r=b, s=A r$, \\
$\rho=r^{T} s, p=r, q=s$ \\
Repeat \\
$\quad(q=A p)$ \\
$\quad \alpha=\rho /\|q\|^{2}$ \\
$\quad x \leftarrow x+\alpha p$ \\
$r \leftarrow r-\alpha q$ \\
$\quad s=A r$ \\
$\quad \bar{\rho}=\rho, \rho=r^{T} s$ \\
$\beta=\rho / \bar{\rho}$ \\
$p \leftarrow r+\beta p$ \\
$q \leftarrow s+\beta q$ \\
\hline
\end{tabular}

\begin{tabular}{l}
$\mathrm{CR}$ \\
\hline Initialize \\
$x_{0}=0, r_{0}=b, s_{0}=A r_{0}$, \\
$\rho_{0}=r_{0}^{T} s_{0}, p_{0}=r_{0}, q_{0}=s_{0}$
\end{tabular}

For $k=1,2, \ldots$

$$
\begin{aligned}
& \left(q_{k-1}=A p_{k-1}\right) \\
& \alpha_{k}=\rho_{k-1} /\left\|q_{k-1}\right\|^{2} \\
& x_{k}=x_{k-1}+\alpha_{k} p_{k-1} \\
& r_{k}=r_{k-1}-\alpha_{k} q_{k-1} \\
& s_{k}=A r_{k} \\
& \rho_{k}=r_{k}^{T} s_{k} \\
& \beta_{k}=\rho_{k} / \rho_{k-1} \\
& p_{k}=r_{k}+\beta_{k} p_{k-1} \\
& q_{k}=s_{k}+\beta_{k} q_{k-1}
\end{aligned}
$$

We are now able to prove our main theorem about the monotonic increase of $\left\|x_{k}\right\|$ for CR and MINRES. A similar result was proved for CG by Steihaug (1983).

Theorem 2.3 For CR (and hence MINRES) on an spd system $A x=b,\left\|x_{k}\right\|$ increases monotonically.

Proof. $\left\|x_{i}\right\|^{2}-\left\|x_{i-1}\right\|^{2}=2 \alpha_{i} x_{i-1}^{T} p_{i-1}+p_{i-1}^{T} p_{i-1} \geq 0$, where the last inequality follows from Theorem 2.2(a), (d) and (e). Therefore $\left\|x_{i}\right\| \geq\left\|x_{i-1}\right\|$.

$\left\|x *-x_{k}\right\|$ is known to be monotonic for CG (Hestenes and Stiefel, 1952). The corresponding result for CR is a direct consequence of (Hestenes and Stiefel, 1952, Theorem 7.5). However, the second half of that theorem, $\left\|x *-x_{k-1}^{C}\right\|>\left\|x *-x_{k}^{M}\right\|$, rarely holds in machine arithmetic. We give here an alternative proof that does not depend on CG. 


\section{CG VERSUS MINRES: AN EMPIRICAL COMPARISON}

Theorem 2.4 For CR (and hence MINRES) on an spd system $A x=b$, the error $\left\|x *_{-} x_{k}\right\|$ decreases monotonically.

Proof. From the update rule for $x_{k}$, we can express the final solution $x_{l}=x *$ as

$$
\begin{aligned}
x_{l} & =x_{l-1}+\alpha_{l-1} p_{l-1} \\
& =\cdots \\
& =x_{k}+\alpha_{k+1} p_{k}+\cdots+\alpha_{l-1} p_{l-1} \\
& =x_{k-1}+\alpha_{k} p_{k-1}+\alpha_{k+1} p_{k}+\cdots+\alpha_{l-1} p_{l-1} .
\end{aligned}
$$

Using equations (3) and (4), we can write

$$
\begin{aligned}
\left\|x_{l}-x_{k-1}\right\|^{2}-\left\|x_{l}-x_{k}\right\|^{2} & =\left(x_{l}-x_{k-1}\right)^{T}\left(x_{l}-x_{k-1}\right)-\left(x_{l}-x_{k}\right)^{T}\left(x_{l}-x_{k}\right) \\
& =2 \alpha_{k} p_{k-1}^{T}\left(\alpha_{k+1} p_{k}+\cdots+\alpha_{l-1} p_{l-1}\right)+\alpha_{k}^{2} p_{k-1}^{T} p_{k-1} \\
& \geq 0,
\end{aligned}
$$

where the last inequality follows from Theorem 2.2 (a), (d).

The energy norm error $\left\|x *-x_{k}\right\|_{A}$ is monotonic for CG by design. The corresponding result for CR is given in (Hestenes and Stiefel, 1952, Theorem 7.4). We give an alternative proof here.

Theorem 2.5 For CR (and hence MINRES) on an spd system $A x=b$, the error in energy norm $\left\|x *-x_{k}\right\|_{A}$ is strictly decreasing.

Proof. From (3) and (4), we can write

$$
\begin{aligned}
\left\|x_{l}-x_{k-1}\right\|_{A}^{2} & -\left\|x_{l}-x_{k}\right\|_{A}^{2} \\
& =\left(x_{l}-x_{k-1}\right)^{T} A\left(x_{l}-x_{k-1}\right)-\left(x_{l}-x_{k}\right)^{T} A\left(x_{l}-x_{k}\right) \\
& =2 \alpha_{k} p_{k-1}^{T} A\left(\alpha_{k+1} p_{k}+\cdots+\alpha_{l-1} p_{l-1}\right)+\alpha_{k}^{2} p_{k-1}^{T} A p_{k-1} \\
& =2 \alpha_{k} q_{k-1}^{T}\left(\alpha_{k+1} p_{k}+\cdots+\alpha_{l-1} p_{l-1}\right)+\alpha_{k}^{2} q_{k-1}^{T} p_{k-1} \\
& >0,
\end{aligned}
$$

where the last inequality follows from Theorem 2.2 (a), (c).

\section{Backward error analysis}

For many physical problems requiring numerical solution, we are given inexact or uncertain input data (in this case $A$ and/or $b$ ). It is not justifiable to seek a solution beyond the accuracy of the data (Dongarra et al., 1979). Instead, it is more reasonable to stop an iterative solver once we know that the current approximate solution solves a nearby problem. The measure of "nearby" should match the error in the input data. The design of such stopping rules is an important application of backward error analysis.

For a consistent linear system $A x=b$, we think of $x_{k}$ coming from the $k$ th iteration of one of the iterative solvers. Following Titley-Péloquin (2010) we say that $x_{k}$ is an acceptable solution if and only if there exist perturbations $E$ and $f$ satisfying

$$
(A+E) x_{k}=b+f, \quad \frac{E}{A} \leq \alpha, \quad \frac{f}{b} \leq \beta
$$

for some tolerances $\alpha \geq 0, \beta \geq 0$ that reflect the (preferably known) accuracy of the data. We are naturally 


\section{DAVID CHIN-LUNG FONG and MICHAEL SAUNDERS}

interested in minimizing the size of $E$ and $f$. If we define the optimization problem

$$
\min _{\xi, E, f} \xi \quad \text { s.t. }(A+E) x_{k}=b+f, \quad \frac{\|E\|}{\|A\|} \leq \alpha \xi, \quad \frac{\|f\|}{\|b\|} \leq \beta \xi
$$

to have optimal solution $\xi_{k}, E_{k}, f_{k}$ (all functions of $x_{k}, \alpha$, and $\beta$ ), we see that $x_{k}$ is an acceptable solution if and only if $\xi_{k} \leq 1$. We call $\xi_{k}$ the normwise relative backward error (NRBE) for $x_{k}$.

With $r_{k}=b-A x_{k}$, the optimal solution $\xi_{k}, E_{k}, f_{k}$ is shown in (Titley-Péloquin, 2010) to be

$$
\begin{array}{ll}
\phi_{k}=\frac{\beta\|b\|}{\alpha\|A\|\left\|x_{k}\right\|+\beta\|b\|}, & E_{k}=\frac{\left(1-\phi_{k}\right)}{\left\|x_{k}\right\|^{2}} r_{k} x_{k}^{T}, \\
\xi_{k}=\frac{\left\|r_{k}\right\|}{\alpha\|A\|\left\|x_{k}\right\|+\beta\|b\|}, & f_{k}=-\phi_{k} r_{k} .
\end{array}
$$

See (Higham, 2002, p12) for the case $\beta=0$ and (Higham, 2002, §7.1 and p336) for the case $\alpha=\beta$.

\subsection{Stopping rule}

For general tolerances $\alpha$ and $\beta$, the condition $\xi_{k} \leq 1$ for $x_{k}$ to be an acceptable solution becomes

$$
\left\|r_{k}\right\| \leq \alpha\|A\|\left\|x_{k}\right\|+\beta\|b\|,
$$

the stopping rule used in LSQR for consistent systems (Paige and Saunders, 1982a, p54, rule S1).

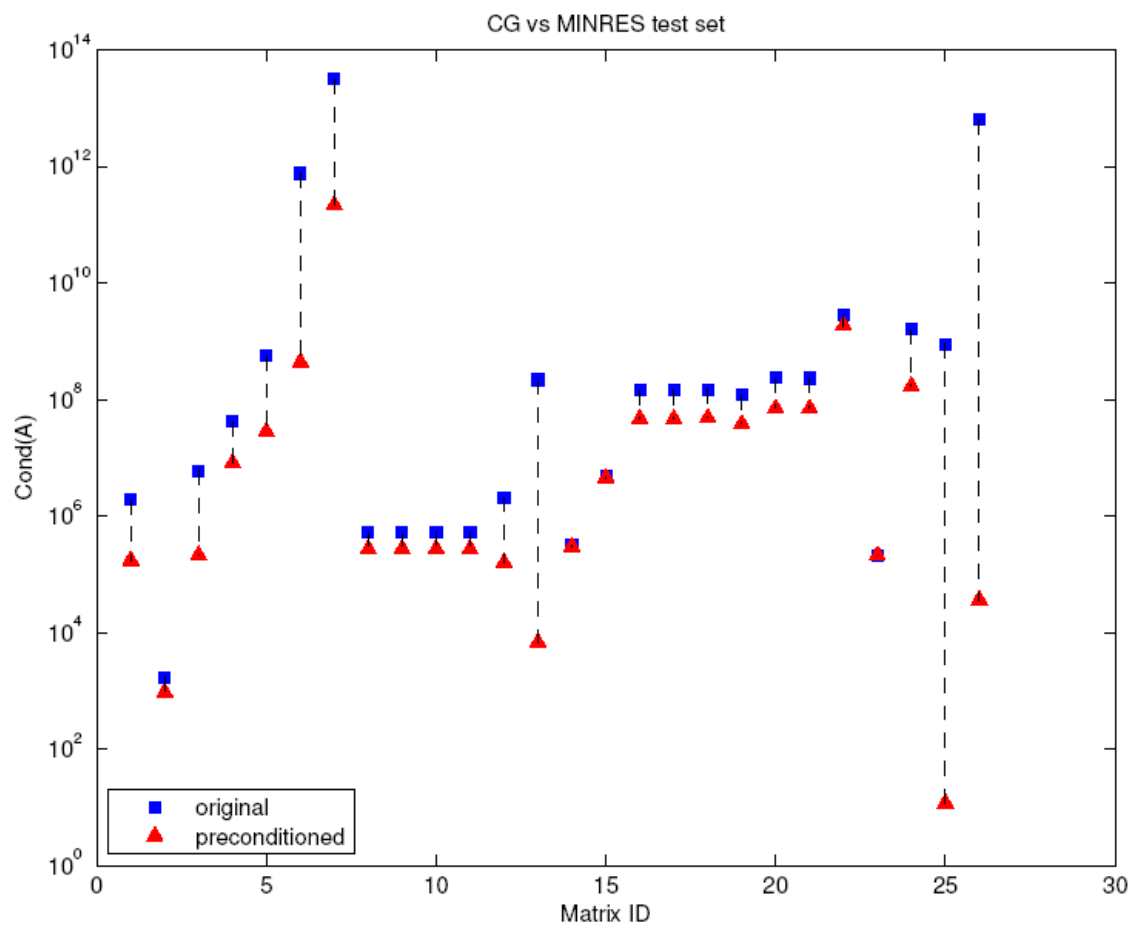

F

igure 1. Comparison of distribution of the condition number for matrices used for CG vs MINRES, before and after diagonal preconditioning. 


\section{CG VERSUS MINRES: AN EMPIRICAL COMPARISON}

\subsection{Monotonic backward errors}

Of interest is the size of the perturbations to $A$ and $b$ for which $x_{k}$ is an exact solution of $A x=b$. From (6)-(7), the perturbations have the following norms:

$$
\begin{aligned}
& \left\|E_{k}\right\|=\left(1-\phi_{k}\right) \frac{\left\|r_{k}\right\|}{\left\|x_{k}\right\|}=\frac{\alpha\|A\|\left\|r_{k}\right\|}{\alpha\|A\|\left\|x_{k}\right\|+\beta\|b\|}, \\
& \left\|f_{k}\right\|=\phi_{k}\left\|r_{k}\right\|=\frac{\beta\|b\|\left\|r_{k}\right\|}{\alpha\|A\|\left\|x_{k}\right\|+\beta\|b\|} .
\end{aligned}
$$

Since $\left\|x_{k}\right\|$ is monotonically increasing for CG and MINRES, we see from (6) that $\phi_{k}$ is monotonically decreasing for both solvers. Since $\left\|r_{k}\right\|$ is monotonically decreasing for MINRES (but not for CG), we have the following result.

Theorem 3.1 Suppose $\alpha>0$ and $\beta>0$ in (5). For CR and MINRES (but not CG), the relative backward errors $\left\|E_{k}\right\| /\|A\|$ and $\left\|f_{k}\right\| /\|b\|$ decrease monotonically.

Proof. This follows from (9)-(10) with $\left\|x_{k}\right\|$ increasing for both solvers and $\left\|r_{k}\right\|$ decreasing for CR and MINRES but not for CG.

\section{Numerical results}

Here we compare the convergence of CG and MINRES on various spd systems $A x=b$ and some associated indefinite systems $(A-\delta I) x=b$. The test examples are drawn from the University of Florida Sparse Matrix Collection (Davis, 2007). We experimented with all 26 cases for which $A$ is real spd and $b$ is supplied. In Matlab we computed the condition number for each test matrix by finding the largest and smallest eigenvalue using eigs $(A, 1, ' L M ')$ and eigs $(A, 1, ' S M ')$ respectively. For this test set, the condition numbers range from $1.7 \mathrm{E}+03$ to $3.1 \mathrm{E}+13$.

Since $A$ is spd, we apply diagonal preconditioning by redefining $A$ and $b$ as follows: $d=\operatorname{diag}(A)$, $D=\operatorname{diag}(1 . / \operatorname{sqrt}(d)), A \leftarrow D A D, b \leftarrow D b, b \leftarrow b /\|b\|$. Thus in the figures below we have $\operatorname{diag}(A)=I$ and $\|b\|=1$. With this preconditioning, the condition numbers range from $1.2 \mathrm{E}+01$ to $2.2 \mathrm{E}+11$. The distribution of the condition number of the test set matrices before and after preconditioning is shown in Figure 1.

The stopping rule used for CG and MINRES was (8) with $\alpha=0$ and $\beta=10^{-8}$. That is, $\left\|r_{k}\right\| \leq 10^{-8}\|b\|=10^{-8}$ (but with a maximum of $5 n$ iterations for spd systems and $n$ iterations for indefinite systems).

\subsection{Positive-definite systems}

In plotting backward errors, we assume for simplicity that $\alpha>0$ and $\beta=0$ in (5)-(7), even though it doesn't match the choice of $\alpha$ and $\beta$ in the stopping rule (8). This gives $\phi_{k}=0$ and $\left\|E_{k}\right\|=\left\|r_{k}\right\| /\left\|x_{k}\right\|$ in (9). Thus, as in Theorem 3.1, we expect $\left\|E_{k}\right\|$ to decrease monotonically for CR and MINRES but not for CG. 
DAVID CHIN-LUNG FONG and MICHAEL SAUNDERS
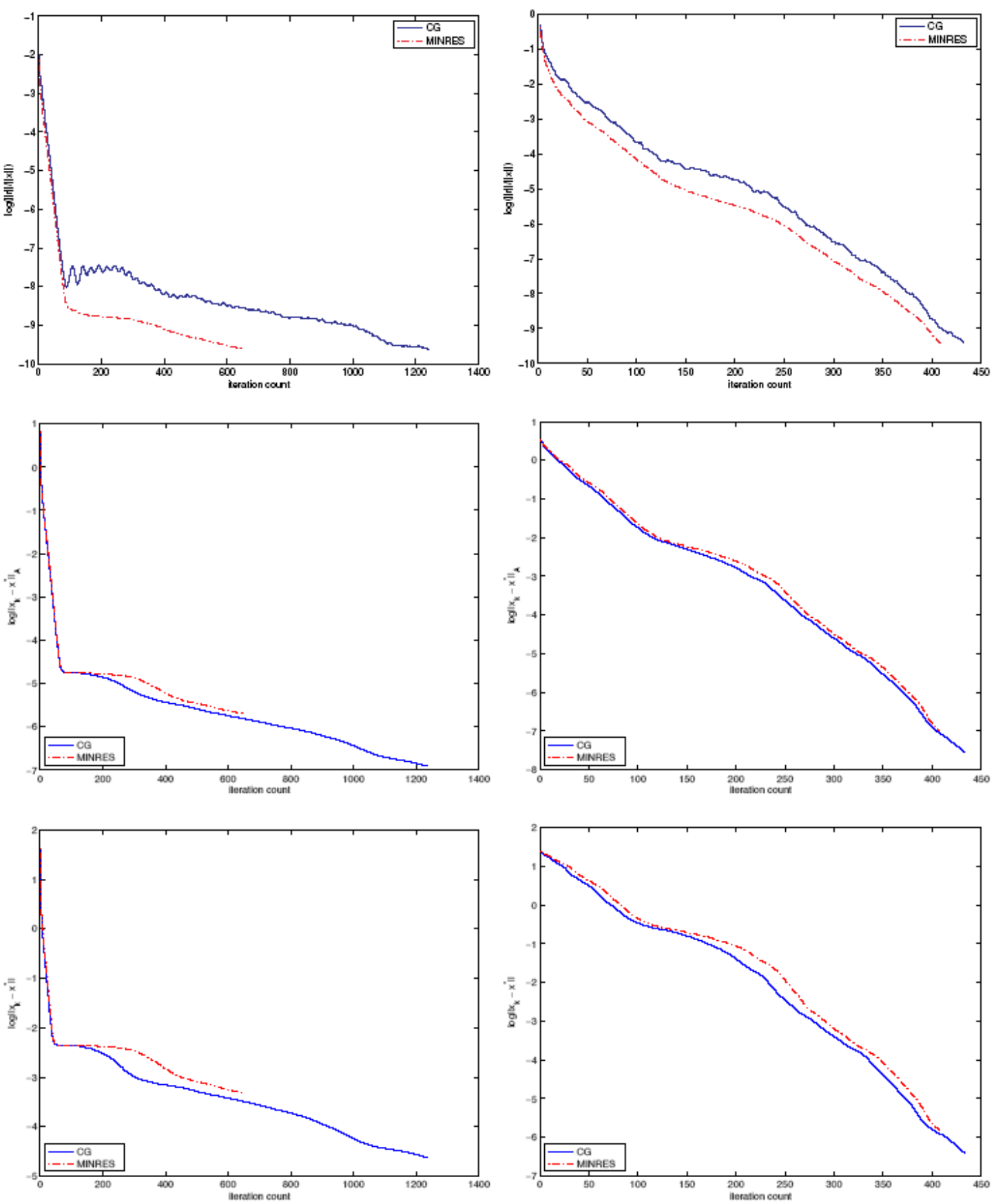

Figure 2. Comparison of backward and forward errors for CG and MINRES solving two spd systems $A x=b$. 


\section{CG VERSUS MINRES: AN EMPIRICAL COMPARISON}
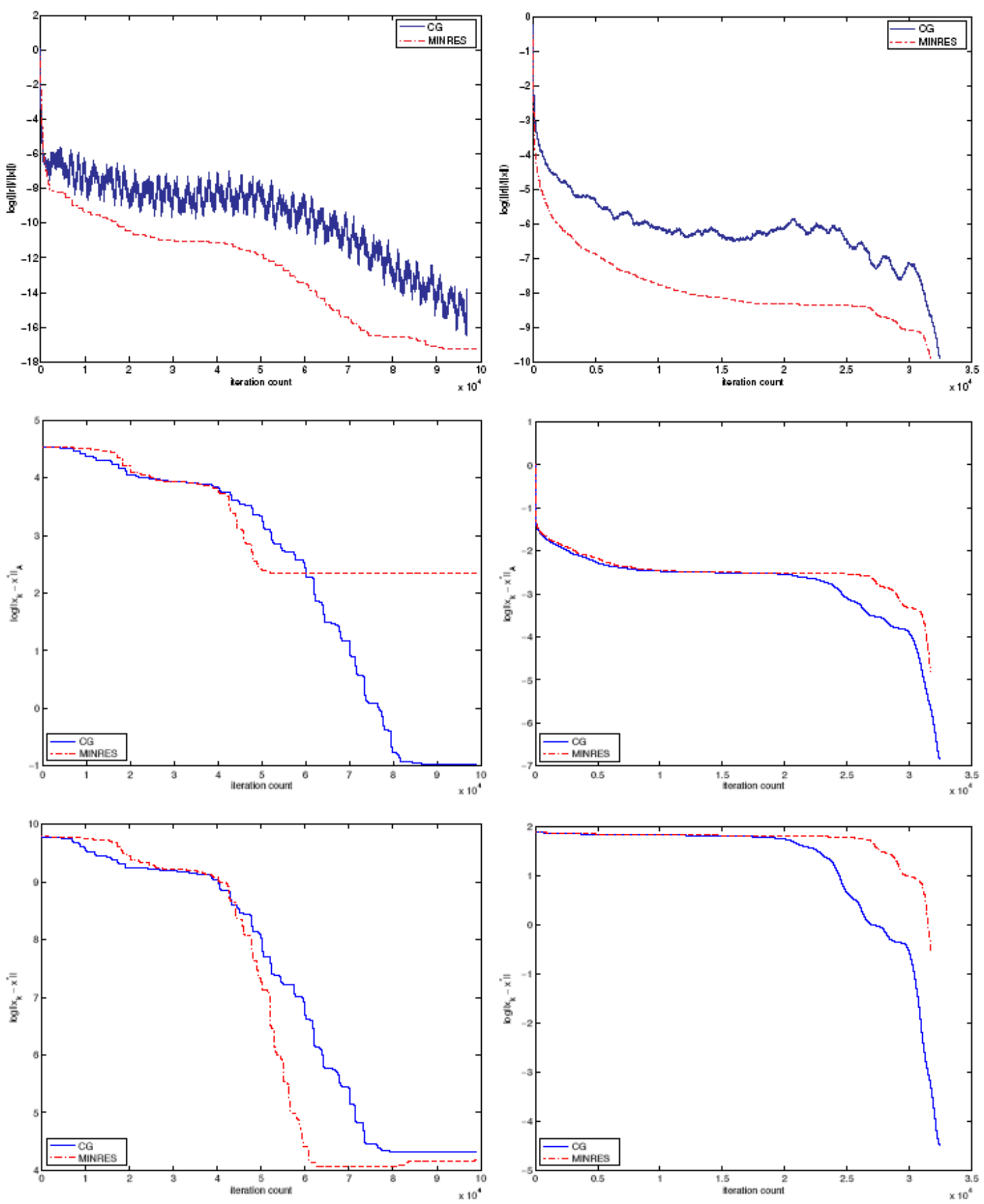

Figure 3. Comparison of backward and forward errors for CG and MINRES solving two more spd systems $A x=b$. 


\section{DAVID CHIN-LUNG FONG and MICHAEL SAUNDERS}

In Figures 2 and 3, we plot $\left\|r_{k}\right\| /\left\|x_{k}\right\|,\left\|x *-x_{k}\right\|_{A}$ (the quantity that CG minimizes at each iteration), and $\left\|x^{*}-x_{k}\right\|$ against iteration number $k$ for CG and MINRES for four different problems. For CG, the plots confirm that $\left\|x *-x_{k}\right\|_{A}$ and $\left\|x *-x_{k}\right\|$ are monotonic. For MINRES, the plots confirm the prediction of Theorems 3.1, 2.5, and 2.4, that $\left\|r_{k}\right\| /\left\|x_{k}\right\|,\left\|x *_{-} x_{k}\right\|_{A}$, and $\left\|x *_{-} x_{k}\right\|$ are monotonic.

Figure 2 (left) shows problem Schenk_AFE_af_shell8 with $A$ of size $504855 \times 504855$ and cond $(A)=$ 2.7E+05 (MINRES stops significantly sooner than CG). From the plot of backward errors $\left\|r_{k}\right\| /\left\|x_{k}\right\|$, we see that both CG and MINRES converge quickly at the early iterations. Then the backward error of MINRES plateaus at about iteration 80, and the backward error of CG stays about 1 order of magnitude behind MINRES. A similar phenomenon of fast convergence at early iterations followed by slow convergence is observed in the energy norm error and 2-norm error plots.

Figure 2 (right) shows problem Cannizzo_sts4098 with $A$ of size $4098 \times 4098$ and $\operatorname{cond}(A)=6.7 \mathrm{E}+03$ (MINRES stops slightly sooner than CG). MINRES converges slightly faster in terms of backward error, while CG converges slightly faster in terms of both error norms.
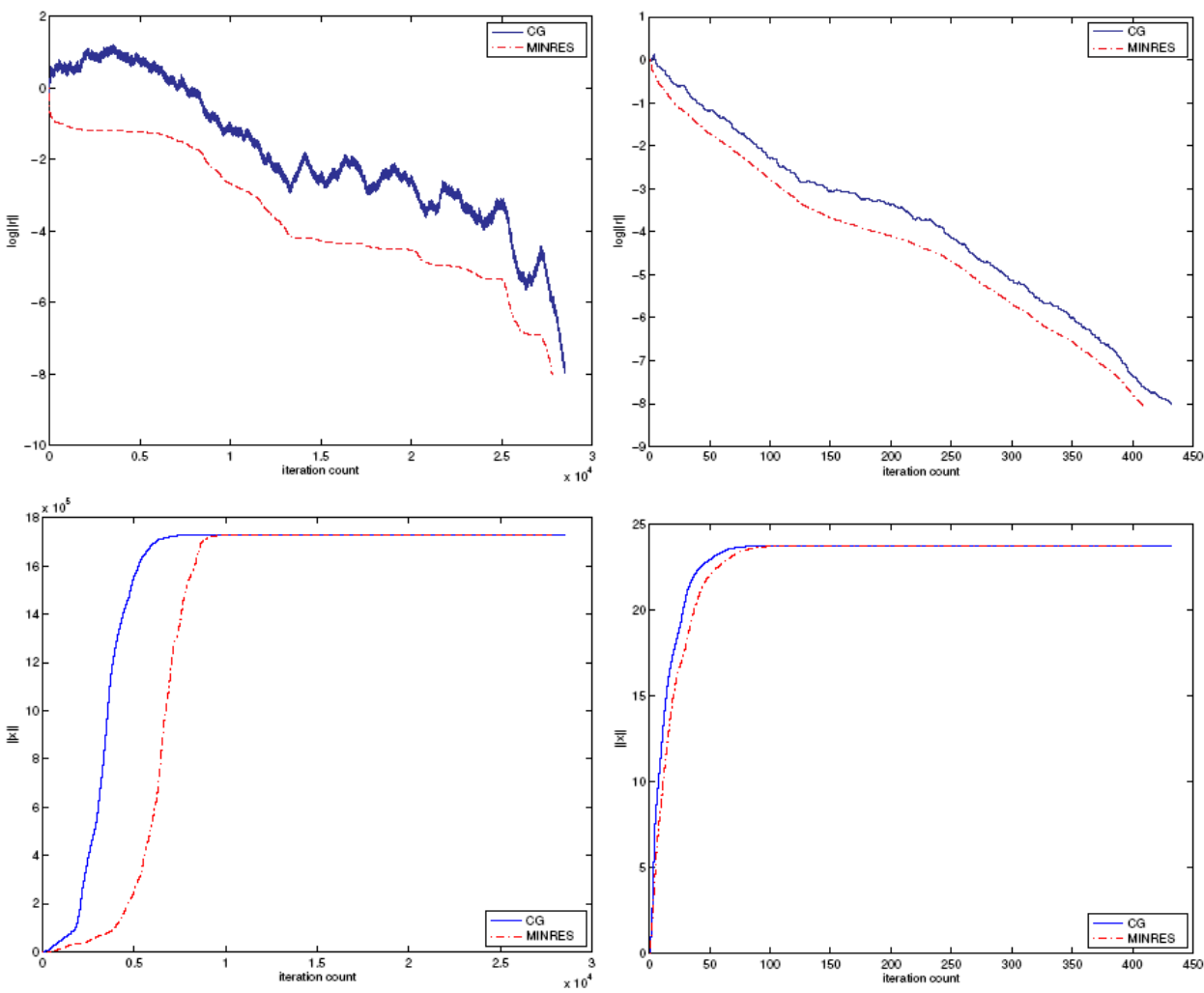

Figure 4. Comparison of residual and solution norms for CG and MINRES solving two spd systems $A x=b$.

Figure 3 (left) shows problem Simon_raefsky4 with $A$ of size $19779 \times 19779$ and $\operatorname{cond}(A)=2.2 \mathrm{E}+11$. Because of the high condition number, both algorithms hit the $5 n$ iteration limit. We see that the backward error for MINRES converges faster than for CG as expected. For the energy norm error, CG is able to decrease over 5 orders of magnitude while MINRES plateaus after a 2 orders of magnitude decrease. For both the energy norm 


\section{CG VERSUS MINRES: AN EMPIRICAL COMPARISON}

error and 2-norm error, MINRES reaches a lower point than CG for some iterations. This must be due to numerical error in CG and MINRES (a result of loss of orthogonality in $V_{k}$ ).

Figure 3 (right) shows problem BenElechi_BenElechi1 with $A$ of size $245874 \times 245874$ and cond $(A)=$ 1.8E+09. The backward error of MINRES stays ahead of CG by 2 orders of magnitude for most iterations. Around iteration 32000, the backward error of both algorithms goes down rapidly and CG catches up with MINRES. Both algorithms exhibit a plateau on energy norm error for the first 20000 iterations. The error norms for CG start decreasing around iteration 20000 and decrease even faster after iteration 30000.

Figures 4 and 5 show $\left\|r_{k}\right\|$ and $\left\|x_{k}\right\|$ against iteration number $k$ for CG and MINRES on four different problems. The solution norms grow somewhat faster for CG than for MINRES. Both reach the limiting value $\|x *\|$ significantly before $x_{k}$ is close to $x *$. Figure 4 (left) shows problem Simon_olafu with $A$ of size $16146 \times 16146$ and $\operatorname{cond}(A)=4.3 \mathrm{E}+08$, and (right) shows problem Cannizzo_sts4098 with $A$ of size $4098 \times 4098$ and $\operatorname{cond}(A)=6.7 \mathrm{E}+03$. We see that $\left\|x_{k}\right\|$ is monotonically increasing for both solvers, and the $\left\|x_{k}\right\|$ values rise fairly rapidly to their limiting value, with a moderate delay for MINRES.
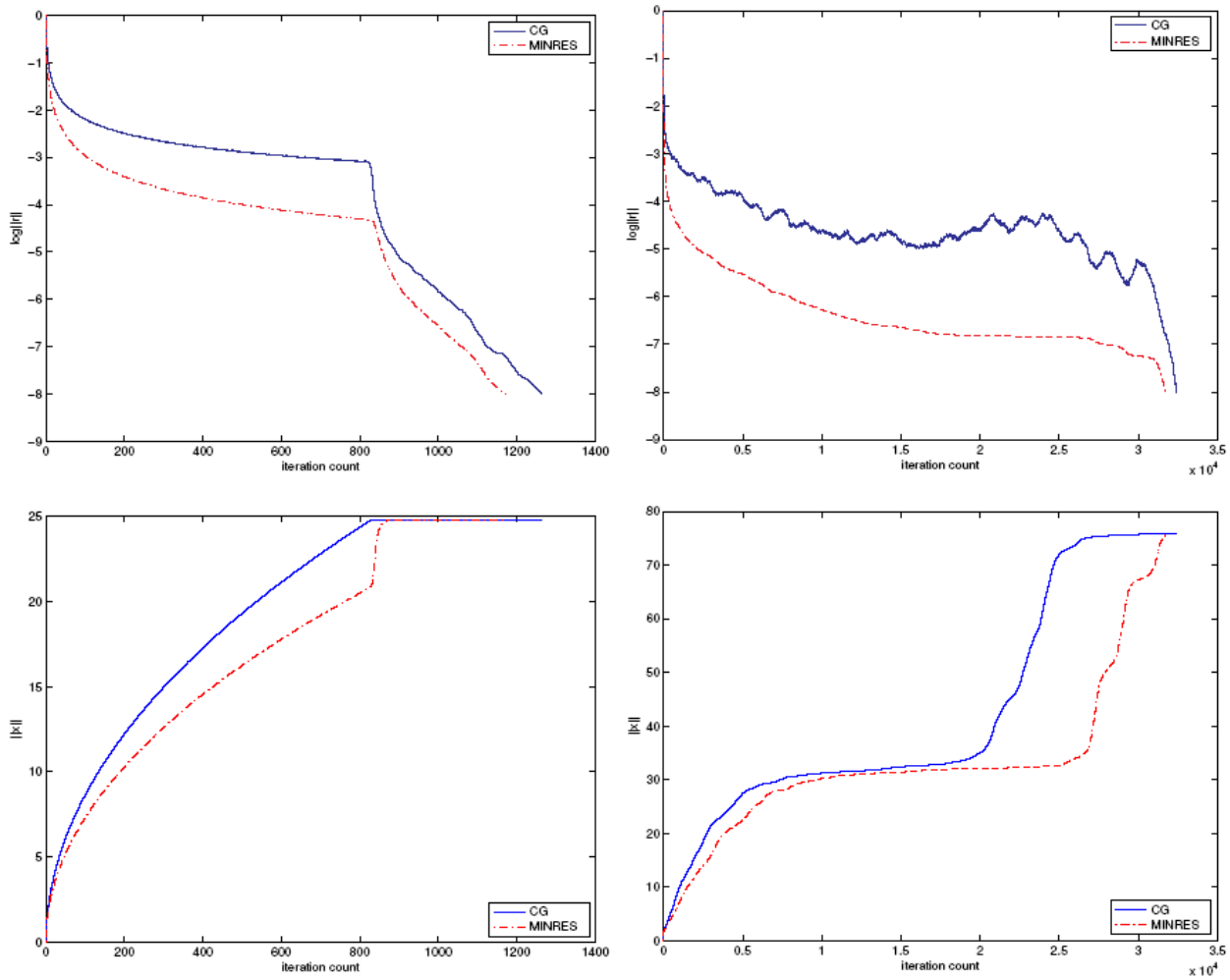

Figure 5. Comparison of residual and solution norms for CG and MINRES solving two more spd systems $A x=b$.

Figure 5 (left) shows problem Schmid_thermal1 with $A$ of size $82654 \times 82654$ and $\operatorname{cond}(A)=3.0 \mathrm{E}+05$, and (right) shows problem BenElechi_BenElechi1 with $A$ of size $245874 \times 245874$ and $\operatorname{cond}(A)=1.8 \mathrm{E}+09$, in which the residual decrease and the solution norm increase are somewhat slower than typical. The rise of $\left\|x_{k}\right\|$ 


\section{DAVID CHIN-LUNG FONG and MICHAEL SAUNDERS}

for MINRES is rather more delayed. In the second case, if the stopping tolerance were $\beta=10^{-6}$ rather than $\beta=10^{-8}$, the final MINRES $\left\|x_{k}\right\|(k \approx 10000)$ would be less than half the exact value $\|x *\|$. It will be of future interest to evaluate this effect within the context of trust-region methods for optimization.

\subsubsection{Why does $r_{k}$ for CG lag behind MINRES?}

It is commonly thought that even though MINRES is known to minimize $\left\|r_{k}\right\|$ at each iteration, the cumulative minimum of $\left\|r_{k}\right\|$ for CG should approximately match that of MINRES. That is,

$$
\min _{0 \leq i \leq k}\left\|r_{i}^{C}\right\| \approx\left\|r_{k}^{M}\right\| \text {. }
$$

However, in Figures 2 and 3 we see that $\left\|r_{k}\right\|$ for MINRES is often smaller than for CG by 1 or 2 orders of magnitude. This phenomenon can be explained by the following relations between $\left\|r_{k}^{C}\right\|$ and $\left\|r_{k}^{M}\right\|$ (Greenbaum, 1977, Lemma 5.4.1; Titley-Peloquin, 2011):

$$
\left\|r_{k}^{C}\right\|=\frac{\left\|r_{k}^{M}\right\|}{\sqrt{1-\left\|r_{k}^{M}\right\|^{2} /\left\|r_{k-1}^{M}\right\|^{2}}} .
$$

From (11), one can infer that if $\left\|r_{k}^{M}\right\|$ decreases a lot between iterations $k-1$ and $k$, then $\left\|r_{k}^{C}\right\|$ would be roughly the same as $\left\|r_{k}^{M}\right\|$. The converse also holds, in that $\left\|r_{k}^{C}\right\|$ will be much larger than $\left\|r_{k}^{M}\right\|$ if MINRES is almost stalling at iteration $k$ (i.e., $\left\|r_{k}^{M}\right\|$ did not decrease much relative to the previous iteration). The above analysis was pointed out by Titley-Peloquin (2011) in comparing LSQR and LSMR (Fong and Saunders, 2011). We repeat the analysis here for CG vs MINRES and extend it to demonstrate why there is a lag in general for large problems.

With $\alpha=0$ in stopping rule (8), CG and MINRES stop when $\left\|r_{k}\right\| \leq \beta\|b\|$. If this occurs at iteration $l$, we have

$$
\prod_{k=1}^{l} \frac{\left\|r_{k}\right\|}{\left\|r_{k-1}\right\|}=\frac{\left\|r_{l}\right\|}{\|b\|} \approx \beta
$$

Thus on average, $\left\|r_{k}^{M}\right\| /\left\|r_{k-1}^{M}\right\|$ will be closer to 1 if $l$ is large. This means that the larger $l$ is (in absolute terms), the more CG will lag behind MINRES (a bigger gap between $\left\|r_{k}^{C}\right\|$ and $\left\|r_{k}^{M}\right\|$ ).

\subsection{Indefinite systems}

A key part of Steihaug's trust-region method for large-scale unconstrained optimization (Steihaug, 1983) (see also (Conn et al., 2000)) is his proof that when CG is applied to a symmetric (possibly indefinite) system $A x=b$, the solution norms $x_{1}, \cdots, x_{k}$ are strictly increasing as long as $p_{j}^{T} A p_{j}>0$ for all iterations $1 \leq j \leq k$. (We are using the same notation as given in Table 1.)

From our proof of Theorem 2.2, we see that the same property holds for CR and MINRES as long as both $p_{j}^{T} A p_{j}>0$ and $r_{j}^{T} A r_{j}>0$ for all iterations $1 \leq j \leq k$. In case future research finds that MINRES is a useful 


\section{CG VERSUS MINRES: AN EMPIRICAL COMPARISON}

solver in the trust-region context, it is of interest now to offer some empirical results about the behavior of $\left\|x_{k}\right\|$ when MINRES is applied to indefinite systems.

First, on the nonsingular indefinite system

$$
\left(\begin{array}{lll}
2 & 1 & 1 \\
1 & 0 & 1 \\
1 & 1 & 2
\end{array}\right) x=\left(\begin{array}{l}
0 \\
1 \\
1
\end{array}\right),
$$

MINRES gives non-monotonic solution norms, as shown in the left plot of Figure 6 . The decrease in $\left\|x_{k}\right\|$ implies that the backward errors $\left\|r_{k}\right\| /\left\|x_{k}\right\|$ may not be monotonic, as illustrated in the right plot.
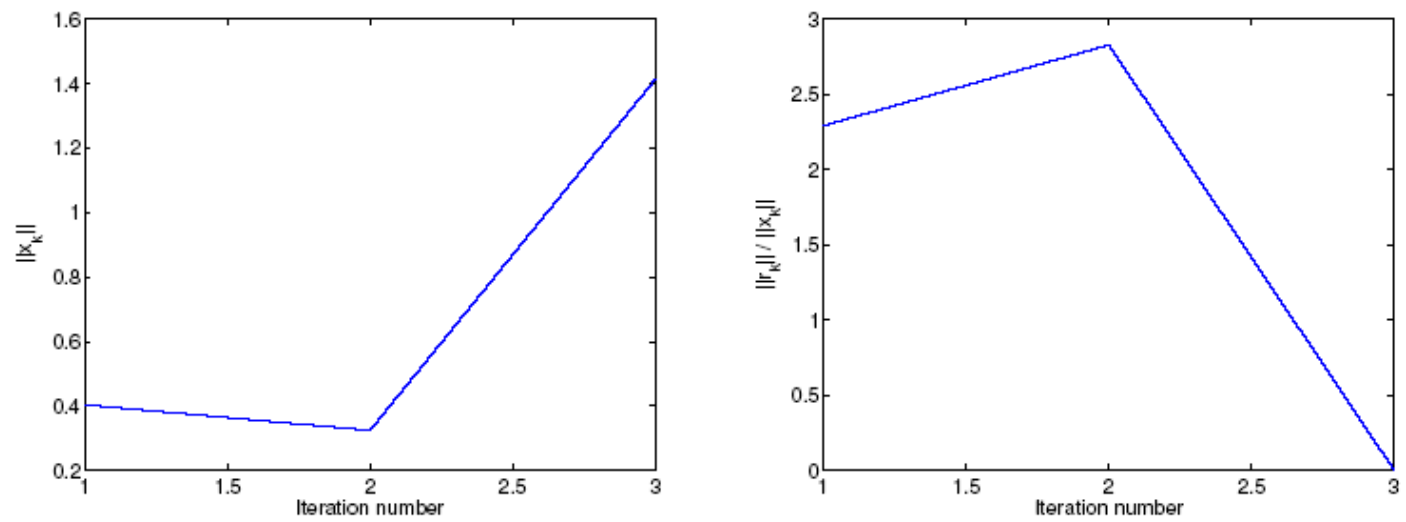

Figure 6. For MINRES on the indefinite problem (4.2), $\left\|x_{k}\right\|$ and the backward error $\left\|r_{k}\right\| /\left\|x_{k}\right\|$ are both slightly non-monotonic.

More generally, we can gain an impression of the behavior of $\left\|x_{k}\right\|$ by recalling from (Choi et al., 2011) the connection between MINRES and MINRES-QLP. Both methods compute the iterates $x_{k}^{M}=V_{k} y_{k}^{M}$ in (1) from the subproblems

$$
y_{k}^{M}=\underset{y \in \mathbb{R}^{k}}{\arg \min }\left\|T_{k} y-\beta_{1} e_{1}\right\| \text { and possibly } T_{\ell} y_{\ell}^{M}=\beta_{1} e_{1} .
$$

When $A$ is nonsingular or $A x=b$ is consistent (which we now assume), $y_{k}^{M}$ is uniquely defined for each $k \leq \ell$ and the methods compute the same iterates $x_{k}^{M}$ (but by different numerical methods). In fact they both compute the expanding QR factorizations

$$
Q_{k}\left[\begin{array}{ll}
T_{k} & \beta_{1} e_{1}
\end{array}\right]=\left[\begin{array}{cc}
R_{k} & t_{k} \\
0 & \phi_{k}
\end{array}\right],
$$

(with $R_{k}$ upper tridiagonal) and MINRES-QLP also computes the orthogonal factorizations $R_{k} P_{k}=L_{k}$ (with $L_{k}$ lower tridiagonal), from which the $k$ th solution estimate is defined by $W_{k}=V_{k} P_{k}, L_{k} u_{k}=t_{k}$, and $x_{k}^{M}=W_{k} u_{k}$. As shown in (Choi et al., $2011 \S 5.3$ ), the construction of these quantities is such that the first $k-3$ columns of $W_{k}$ are the same as in $W_{k-1}$, and the first $k-3$ elements of $u_{k}$ are the same as in $u_{k-1}$. 


\section{DAVID CHIN-LUNG FONG and MICHAEL SAUNDERS}

Since $W_{k}$ has orthonormal columns, $\left\|x_{k}^{M}\right\|=\left\|u_{k}\right\|$, where the first $k-2$ elements of $u_{k}$ are unaltered by later iterations. As shown in (Choi et al., 2011, §6.5), it means that certain quantities can be cheaply updated to give norm estimates in the form

$$
\chi^{2} \leftarrow \chi^{2}+\hat{\mu}_{k-2}^{2}, \quad\left\|x_{k}^{M}\right\|^{2}=\chi^{2}+\tilde{\mu}_{k-1}^{2}+\bar{\mu}_{k}^{2},
$$

where it is clear that $\chi^{2}$ increases monotonically. Although the last two terms are of unpredictable size, $\left\|x_{k}^{M}\right\|^{2}$ tends to be dominated by the monotonic term $\chi^{2}$ and we can expect that $\left\|x_{k}^{M}\right\|$ will be approximately monotonic as $k$ increases from 1 to $\ell$.
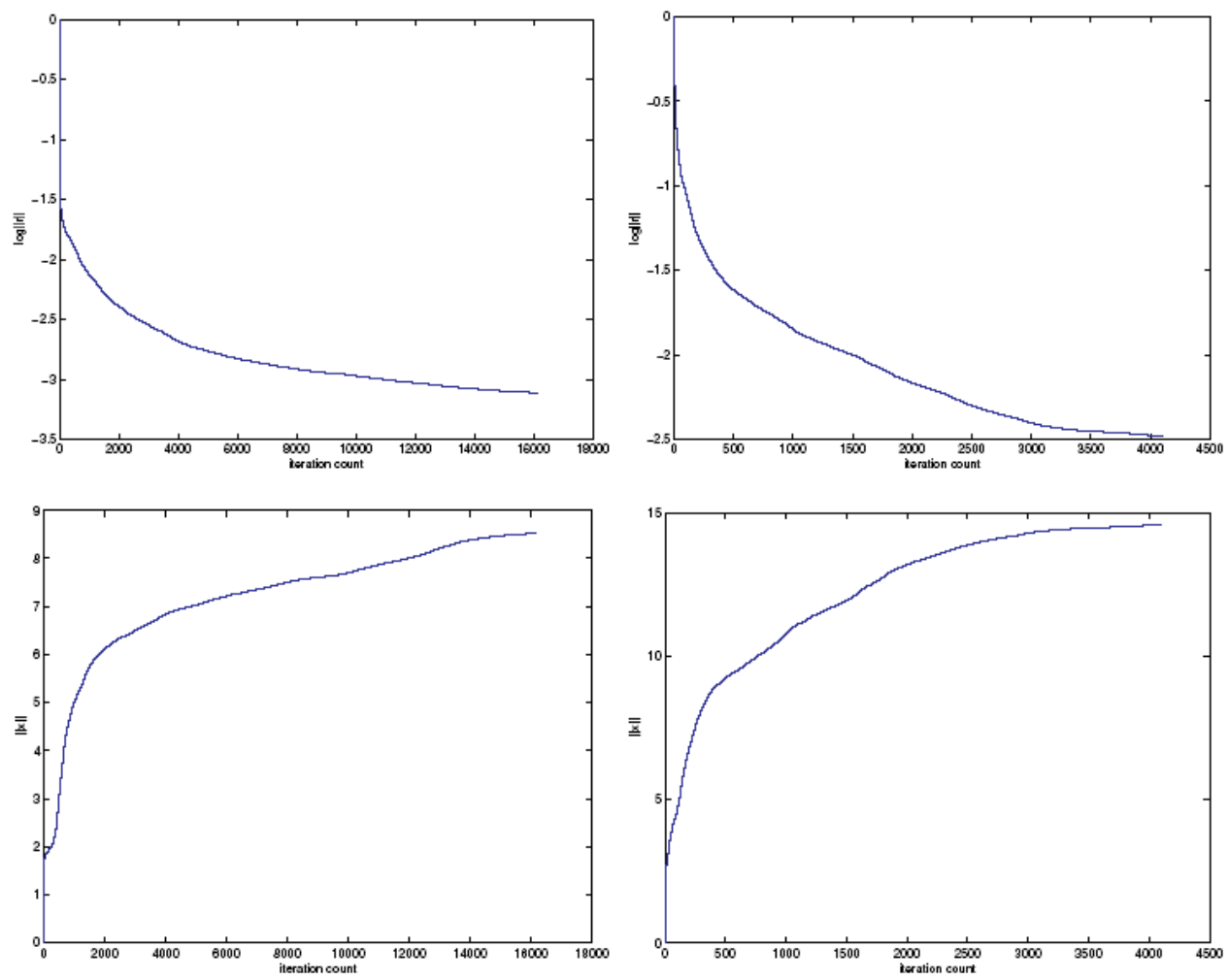

Figure 7. Residual norms and solution norms when MINRES is applied to two indefinite systems $(A-\delta I) x=b$.

Experimentally we find that for most MINRES iterations on an indefinite problem, $\left\|x_{k}\right\|$ does increase. To obtain indefinite examples that were sensibly scaled, we used the four spd $(A, b)$ cases in Figures 4 and 5 , applied diagonal scaling as before, and solved $(A-\delta I) x=b$ with $\delta=0.5$ and where $A$ and $b$ are now scaled (so that $\operatorname{diag}(A)=I$ ). The number of iterations increased significantly but was limited to $n$.

Figures 7 and 8 show residual norms $\log _{10}\left\|r_{k}\right\|$ and and solution norms $\left\|x_{k}\right\|$ against iteration number $k$ 


\section{CG VERSUS MINRES: AN EMPIRICAL COMPARISON}

when MINRES is applied to the four indefinite systems $(A-\delta I) x=b$, where $\delta=0.5$ is large enough to make the systems indefinite.

Figure 7 (left) shows problem Simon_olafu with $n=16146$, and (right) shows problem Cannizzo_sts4098 with $n=4098$ (where $A$ is the spd matrices in Figure 4). The values of $\left\|x_{k}\right\|$ are essentially monotonic. The backward errors $\left\|r_{k}\right\| /\left\|x_{k}\right\|$ (not shown) were even closer to being monotonic (at least for the first $n$ iterations). During the $n=16146$ iterations of Simon_olafu, $\left\|x_{k}\right\|$ increased $83 \%$ of the time and the backward errors $\left\|r_{k}\right\| /\left\|x_{k}\right\|$ (not shown) decreased $96 \%$ of the time; and during the $n=4098$ iterations of Cannizzo_sts4098, $\left\|x_{k}\right\|$ increased $90 \%$ of the time and the backward errors $\left\|r_{k}\right\| /\left\|x_{k}\right\|$ (not shown) decreased $98 \%$ of the time.
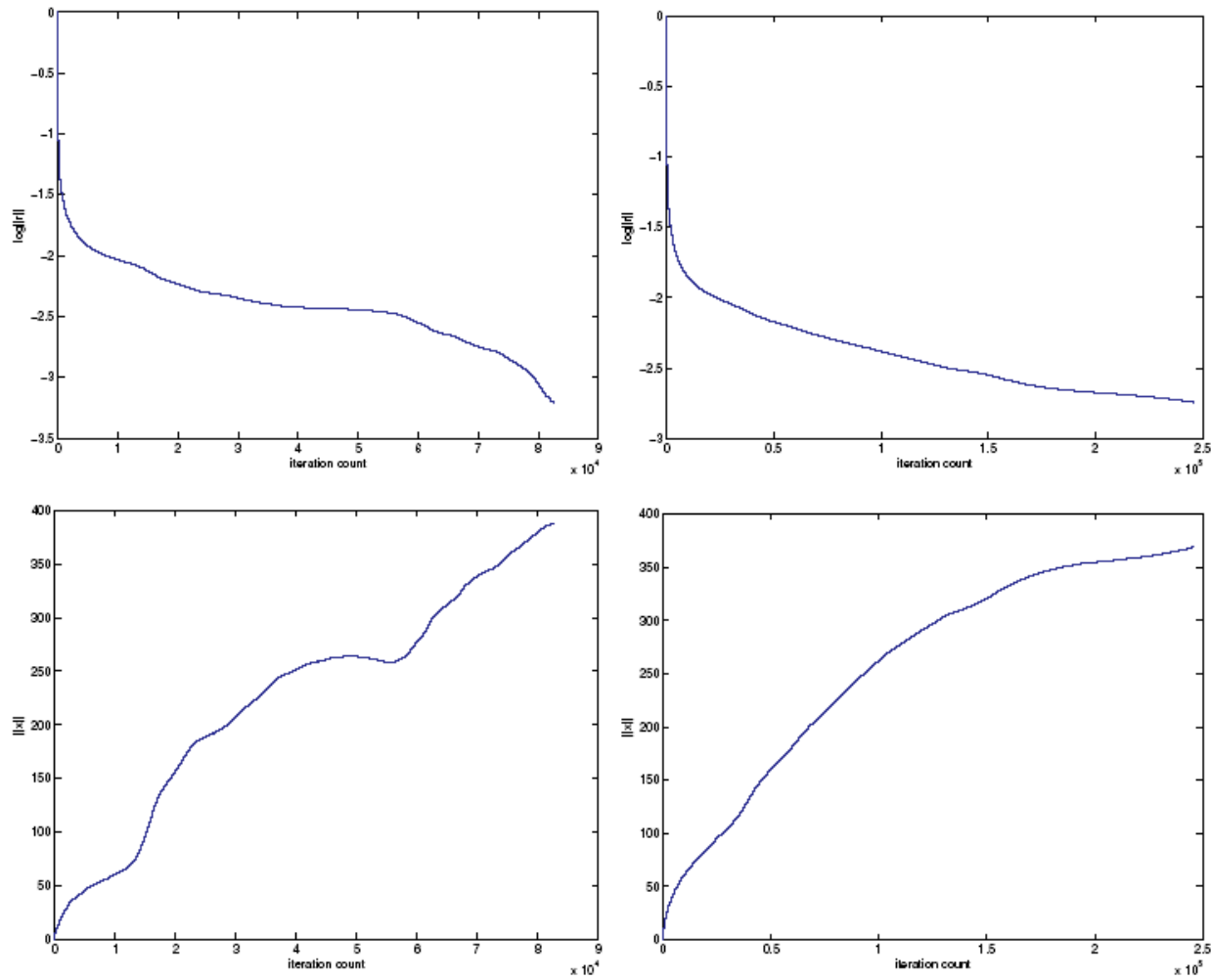

Figure 8. Residual norms and solution norms when MINRES is applied to two more indefinite systems

$$
(A-\delta I) x=b \text {. }
$$

Figure 8 (left) shows problem Schmid_thermal1 with $n=82654$, and (right) shows problem BenElechi_BenElechi1 with $n=245874$ (where $A$ is the spd matrices in Figure 5). The problem Schmid_thermal1 reveals a definite period of decrease in $\left\|x_{k}\right\|$, and there is a mild but clear decrease in $\left\|x_{k}\right\|$ over an interval of about 10000 iterations. Nevertheless, during the $n=82654$ iterations, $\left\|x_{k}\right\|$ increased $83 \%$ of the time and the backward errors $\left\|r_{k}\right\| /\left\|x_{k}\right\|$ decreased $91 \%$ of the time. The problem BenElechi_BenElechi1 is more like those 


\section{DAVID CHIN-LUNG FONG and MICHAEL SAUNDERS}

in Figure 7, where the solution norms and backward errors are essentially monotonic. During the $n=245874$ iterations, $\left\|x_{k}\right\|$ increased $88 \%$ of the time and the backward errors $\left\|r_{k}\right\| /\left\|x_{k}\right\|$ (not shown) decreased $95 \%$ of the time.

\section{Conclusions}

For full-rank least-squares problems $\min \|A x-b\|$, the solvers LSQR (Paige and Saunders, 1982a, 1982b) and LSMR (Fong and Saunders, 2011, 2010) are equivalent to CG and MINRES on the (spd) normal equation $A^{T} A x=A^{T} b$. Comparisons in (Fong and Saunders, 2011) indicated that LSMR can often stop much sooner than LSQR when the stopping rule is based on Stewart's backward error norm $\left\|A^{T} r_{k}\right\| /\left\|r_{k}\right\|$ for least-squares problems (Stewart, 1977).

Table 2. Comparison of CG and MINRES properties on an spd system $A x=b$

\begin{tabular}{lcl} 
& $\mathrm{CG}$ & MINRES \\
\hline$\left\|x_{k}\right\|$ & $\nearrow[21$, Thm 2.1] & $\nearrow($ Thm 2.3) \\
$\left\|x^{*}-x_{k}\right\|$ & $\searrow[11$, Thm 4:3] & $\searrow$ (Thm 2.4) $[11$, Thm 7:5] \\
$\left\|x^{*}-x_{k}\right\|_{A}$ & $\searrow[11$, Thm 6:3] & $\searrow$ (Thm 2.5) [11, Thm 7:4] \\
$\left\|r_{k}\right\|$ & not-monotonic & $\searrow[18][11$, Thm 7:2] \\
$\left\|r_{k}\right\| /\left\|x_{k}\right\|$ & not-monotonic & $\searrow$ (Thm 3.1) \\
\hline \multicolumn{3}{c}{$\nearrow$ monotonically increasing } \\
$\searrow$ monotonically decreasing
\end{tabular}

[11]=Hestenes and Stiefel (1952); [18]=Paige and Saunders (1975); [21]=Steihaug (1983)

Table 3. Comparison of LSQR and LSMR properties on $\min \|A x-b\|$

\begin{tabular}{lcc} 
& LSQR & LSMR \\
\hline$\left\|x_{k}\right\|$ & $\nearrow[7$, Thm 3.3.1] & $\nearrow[7$, Thm 3.3.6] \\
$\left\|x^{*}-x_{k}\right\|$ & $\searrow[7$, Thm 3.3.2] & $\searrow[7$, Thm 3.3.7] \\
$\left\|r^{*}-r_{k}\right\|$ & $\searrow[7$, Thm 3.3.3] & $\searrow[7$, Thm 3.3.8] \\
$\left\|A^{T} r_{k}\right\|$ & not-monotonic & $\searrow[8, \S 3.2]$ \\
$\left\|r_{k}\right\|$ & $\searrow[19, \S 5.1]$ & $\searrow[7$, Thm 3.3.11] \\
\multicolumn{3}{c}{$x_{k}$ converges to minimum-norm $x^{*}$ for singular systems } \\
\hline
\end{tabular}

[7]=Fong (2011); [8]=Fong and Saunders (2011); [19]=Paige and Saunders (1982)

Our theoretical and experimental results here provide analogous evidence that MINRES can often stop much sooner than CG on spd systems when the stopping rule is based on the backward error $\left\|r_{k}\right\| /\left\|x_{k}\right\|$ for $A x=b$ (or the more general backward errors in Theorem 3.1). In some cases, MINRES can converge faster than CG by as much as 2 orders of magnitude (Figure 3). On the other hand, CG converges somewhat faster than MINRES in terms of both $\left\|x *-x_{k}\right\|_{A}$ and $\left\|x *-x_{k}\right\|$ (same figure). For spd systems, Table 2 summarizes properties that were already known by Hestenes and Stiefel (1952) and Steihaug (1983), along with the two additional properties we proved here (Theorems 2.3 and 3.1). 


\section{CG VERSUS MINRES: AN EMPIRICAL COMPARISON}

These theorems and experiments on CG and MINRES are part of the first author's PhD thesis (Fong, 2011), which also discusses LSQR and LSMR and derives some new results for both solvers. Table 3 summarizes the known results for CG and MINRES in (Paige and Saunders, 1982a) and (Fong and Saunders, 2011), respectively, and the newly derived properties for both solvers in (Fong, 2011).

\section{Acknowledgements}

We kindly thank Professor Mehiddin Al-Baali and other colleagues for organizing the Second International Conference on Numerical Analysis and Optimization at Sultan Qaboos University (January 3-6, 2011, Muscat, Sultanate of Oman). Their wish to publish some of the conference papers in a special issue of SQU Journal for Science gave added motivation for this research. We also thank Dr Sou-Cheng Choi for many helpful discussions of the iterative solvers, and Michael Friedlander for a final welcome tip.

The first author (David Fong) was partially supported by a Stanford Graduate Fellowship. The second author (Michael Saunders) was partially supported by Office of Naval Research grant N00014-08-1-0191 and NSF grant DMS-1009005, and by the U.S. Army Research Laboratory, through the Army High Performance Computing Research Center, Cooperative Agreement W911NF-07-0027.

\section{References}

ARIOLI, M. 2004. A stopping criterion for the conjugate gradient algorithm in a finite element method framework. Numerical Mathematics, 97: 1-24.

CHOI, S.-C. 2006. Iterative Methods for Singular Linear Equations and Least-Squares Problems. PhD thesis, Stanford University, Stanford, California, USA.

CHOI, S.-C., PAIGE, C.C. and SAUNDERS, M.A. 2011. MINRES-QLP: a Krylov subspace method for indefinite or singular symmetric systems. SIAM J. Sci. Comput., 33: 1810-1836.

CONN, A.R., GOULD, N.I.M. and TOINT, PH.L. 2000. Trust-Region Methods, vol. 1. SIAM, Philadelphia, USA.

DAVIS, T.A. 2007. University of Florida Sparse Matrix Collection. http://www.cise.ufl.edu/research/sparse/matrices.

DONGARRA, J.J., BUNCH, J.R., MOLER, C.B. and STEWART, G.W. 1979. LINPACK Users' Guide. SIAM, Philadelphia, USA.

FONG, D.C.-L. 2011. Minimum-Residual Methods for Sparse Least-Squares Using Golub-Kahan Bidiagonalization. PhD thesis, Stanford University, Stanford, California, USA.

FONG, D.C.-L. and SAUNDERS, M.A. 2010. LSMR software for linear systems and least squares. http://www.stanford.edu/group/SOL/software.html.

FONG, D.C.-L. and SAUNDERS, M.A. 2011. LSMR: An iterative algorithm for sparse least-squares problems. SIAM Journal on Scientific Computing, 33: 2950-2971.

FREUND, R.W., GOLUB, G.H. and NACHTIGAL, N.M. 1992. Iterative solution of linear systems. Acta Numerica, 1: 57-100.

GREENBAUM, A. 1997. Iterative Methods for Solving Linear Systems. SIAM, Philadelphia, USA.

HESTENES, M.R. and STIEFEL, E. 1952. Methods of conjugate gradients for solving linear systems. Journal of Research of the National Bureau of Standards, 49: 409-436.

HIGHAM, N.J. 2002. Accuracy and Stability of Numerical Algorithms. (second Edition) SIAM, Philadelphia, USA.

LANCZOS, C. 1950. An iteration method for the solution of the eigenvalue problem of linear differential and integral operators, Journal of Research of the National Bureau of Standards, 45: 255-282.

LUENBERGER, D.G. 1969. Hyperbolic pairs in the method of conjugate gradients. SIAM Journal on Applied Mathematics, 17: 1263-1267. 


\section{DAVID CHIN-LUNG FONG and MICHAEL SAUNDERS}

LUENBERGER, D.G. 1970. The conjugate residual method for constrained minimization problems. SIAM Journal on Numerical Analysis, 7: 390-398.

MEURANT, G.A. 2006. The Lanczos and Conjugate Gradient Algorithms: From Theory to Finite Precision Computations, vol. 19 of Software, Environments, and Tools, SIAM, Philadelphia, USA.

PAIGE, C.C. and SAUNDERS, M.A. 1975. Solution of sparse indefinite systems of linear equations. SIAM Journal on Numerical Analysis, 12: 617-629.

PAIGE, C.C. and SAUNDERS, M.A. 1982a. LSQR: An algorithm for sparse linear equations and sparse least squares. ACM Transactions on Mathematical Software, 8: 43-71.

PAIGE, C.C. and SAUNDERS, M.A. 1982b. Algorithm 583; LSQR: Sparse linear equations and least-squares problems. ACM Transactions on Mathematical Software, 8: 195-209.

STEIHAUG, T. 1983. The conjugate gradient method and trust regions in large scale optimization. SIAM Journal on Numerical Analysis, 20: 626-637.

STEWART, G.W. 1977. Research, development and LINPACK, in Mathematical Software III, J.R. RICE, ed., Academic Press, New York, USA, 1-14.

STIEFEL,E. 1955. Relaxationsmethoden bester strategie zur l“osung linearer gleichungssysteme. Comm. Math. Helv., 29: 157-179.

SUN, Y. 2003. The Filter Algorithm for Solving Large-Scale Eigenproblems from Accelerator Simulations. $\mathrm{PhD}$ thesis, Stanford University, Stanford, California, USA.

TITLEY-PELOQUIN, D. 2010. Backward Perturbation Analysis of Least Squares Problems. PhD thesis, School of Computer Science, McGill University, Canada.

TITLEY-PELOQUIN, D. 2011. Convergence of backward error bounds in LSQR and LSMR. Private communication.

VAN DER VORST, H.A. 2003. Iterative Krylov Methods for Large Linear Systems. (1st Edition) Cambridge University Press, Cambridge, UK.

WATKINS, D.S. 2010. Fundamentals of Matrix Computations. (3rd Edition) Pure and Applied Mathematics, Wiley, Hoboken, New Jersey, USA.

Received 20 October 2011

Accepted 29 November 2011 